\title{
Interference Mitigation of Heterogeneous Cognitive Radio Network using Spatial Diversity
}

\author{
R. H. Adekar, A. K. Kureshi
}

\begin{abstract}
The heterogeneous cognitive radio networks are playing the most important role in the future generation wireless networks in order to address the problem of spectrum scarcity and to satisfy the demand of multiple coexistence networks. . In a country like India the deployment of CRN is possible on television network with the help of TV White Spaces (TVWS) as its capacity is quite high due to digital transmission of TV channel. The use of multiple wireless standards such as IEEE 802.11a, IEEE 802.22, IEEE 802.19.1 and many more wireless networks operating in the same frequency band of TVWS creates the coexistence scenario which involves the heterogeneous networks. The interference mitigation is the most important issue in such heterogeneous networks. In literature, the issue of interference mitigation is addressed mainly at the medium access layer; however, very limited work is presented at physical layer. In this paper, an interference mitigation problem of heterogeneous cognitive radio network at physical layer is addressed. The spatial diversity based techniques are proposed to mitigate the interference in heterogeneous CRN. The coexistence of different wireless networks in secondary CRN is considered for analysis. The characterization of aggregate interference is carried out for different interference scenarios. The proposed system outperform for heterogeneous CRN network over TVWS network.
\end{abstract}

Keywords: Bit Error Rate (BER), Cognitive Radio Network (CRN), Heterogeneous Networks, Multiple Input Multiple Output (MIMO), TV White Space (TVWS).

\section{INTRODUCTION}

The next generation wireless systems need to satisfy the higher data rate demands along with the flexibility in operation and cost effective deployment solutions. It led to use of diverse wireless communication technologies and different network configurations. The radio access technology is evolved from GSM, UMTS to the LTE and LTE advanced which provides the wide range of wireless coverage. The wireless network standards from IEEE 802.11a till IEEE802.11ac for Wireless Local Area Network (WLAN) and IEEE 802.16 for Wireless Metropolitan Area Networks (WMANs) are used to cater the need of higher data rates and last mile coverage. These wireless technologies operate on different frequency bands specified by regularity authorities.

Revised Manuscript Received on December 30, 2019.

* Correspondence Author

R. H. Adekar, Research Scholar, National Institute of Electronics \& Information Technology, Dr. Babasaheb Ambedkar Marathwada University, Aurangabad, India. Email: rhadekar@gmail.com

Dr. A. K. Kureshi, Professor in Department of Electronics and Telecommunication Engineering and Director, MMANTC, College of Engineering MANSOORA, Malegaon, India. Email: akkureshi@rediffmail.com

(C) The Authors. Published by Blue Eyes Intelligence Engineering and Sciences Publication (BEIESP). This is an open access article under the CC BY-NC-ND license (http://creativecommons.org/licenses/by-nc-nd/4.0/)
The spectrum scarcity becomes the major issue in the densely populated urban areas where large number of wireless networks is deployed. The ISM band of $2.4 \mathrm{GHz}$ is shared by many heterogeneous wireless communication systems. The coexistence of different heterogeneous systems can effectively improve the frequency utilization of Infrared (IR) frequency band [1]. Both IEEE 802.11 and IEEE 802.16 standards have become widespread throughout the world in license-exempt spectrum specifically in the $5 \mathrm{GHz}$ band [2]. In general, if wireless devices are spatially coexisting, then there is fear of interference to each due to spectral leakage. If diverse wireless devices are coexisting and operates on the same band (free band) the interference will be worst, even leading to totally no communication.

The spectrum scarcity problem is addressed by Joy Mitola [3] who proposed the concept of Cognitive Radio Network (CRN) which supports the dynamic spectrum access. The CRN uses the dedicated licensed spectrum allocated to primary users either in underlay or overlay mode of operation by the secondary users. In a country like India the deployment of CRN is possible on television network with the help of TV White Spaces (TVWS) as its capacity is quite high due to digital transmission of TV channel [4]. The wireless transmission on TVWS is more convenient due to their superior propagation characteristics enabling large coverage area with minimal infrastructure requirements. The use of TVWS initiated new developments in wireless standards which include IEEE 802.22 used in Wireless Regional Area Network (WRAN), 802.11af, ECMA 392 used for personal devices and even IEEE 802.19.1 [5]. The use of these multiple wireless standards operating in the same frequency band of TVWS creates the coexistence scenario which involves the heterogeneous networks. The characteristics of heterogeneous and coexistence of wireless network is common in all unlicensed band including TVWS. The efficient spectrum utilization is achieved by using TVWS network but it gives many challenges while operating in heterogeneous network scenario. These challenges are mainly classified in to three categories as spectrum sensing, spectrum sharing and interference mitigation [6].

The performance degradation for co-existing IEEE802.11 and IEEE802.16 systems is presented in [7] where the analysis is carried out at the medium access control (MAC) layer by means of an event model based simulation tool. The cognitive radio network is used for the co-existence of IEEE802.11b and IEEE 802.16a networks in [8] by using common frequency band. The coexistence of heterogeneous CR networks by considering the TVWS band is presented in [9].

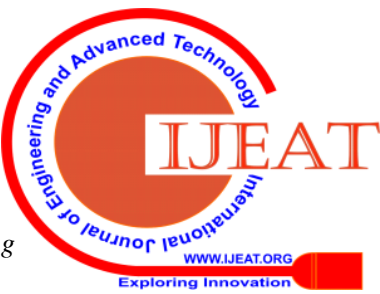




\section{Interference Mitigation of Heterogeneous Cognitive Radio Network using Spatial Diversity}

Here, the mediator system is used to provide the indirect coordination between heterogeneous CR networks. In [10],

the reactive interference avoidance method is presented for heterogeneous networks where adaptively adjusting transmits physical layer parameters are used from the observations of the radio band. The spectrum sharing between coexisting competing wireless systems such as 802.11and 802.16 and characterization of the interference in such network is proposed in [11]. The coexistence of IEEE802.16 (WiMAX) and IEEE802.11 (WiFi) in shared radio spectrum is proposed in [12].

The mechanisms for heterogeneous coexistence in primary and secondary network of CRN are proposed in [13] where the taxonomy of the mechanisms targeting TVWS is discussed. The coexistence of cognitive heterogeneous networks of both primary and secondary nodes is proposed in [14] by using a non-coherent spectrum sensing scheme. The stochastic geometry models for single-tier as well as multi-tier and cognitive cellular wireless networks are presented in [15].

The above literature survey outlines the importance of CRN over TVWS for heterogeneous network. The coexistence of heterogeneous network addresses three types of challenges in spectrum sensing, spectrum sharing and interference mitigation. The spectrum sensing may give a generalized solution for coexistence of multiple diverse wireless technologies. The medium access level frame structure is used commonly by all spectrum sharing networks for exchanging the information. In spectrum dense areas it is very difficult to identify the spectrum holes and the interference mitigation is highly important when there is overlapping of different networks.

All these challenges are addressed in literature mainly at Medium Access Control (MAC) layer. However, very limited work is presented at physical layer. Therefore, this paper proposed an interference mitigation problem of heterogeneous cognitive radio network at physical layer. In this paper the spatial diversity based techniques are proposed to mitigate the interference in heterogeneous CRN. The coexistence of different wireless networks in secondary CRN are considered for analysis. The concept of transmit antenna selection in multiple antenna system and the characterization of interference at secondary heterogeneous CRN network is presented over TVWS network.

\section{SYSTEM MODEL}

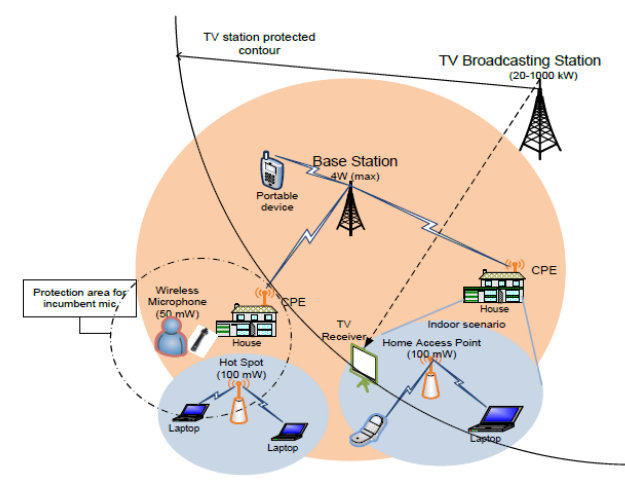

Fig. 1 Heterogeneous Cognitive Radio Network using TVWS
Fig. 1 shows a typical example of heterogeneous CRN using TV broadcasting network with multiple secondary wireless networks. Here, TV broadcasting transmitter is a source of primary network having primary receivers within the protection counter as shown in Fig. 1. The secondary network consists of various wireless networks such as IEEE 802.22 WRANs which consists of Base Stations (BS), fixed CPEs (Consumer Premise Equipment's). It also consists of IEEE 8.2.11a Wi-Fi home networks and hot spots operating in the TVWS. The low power wireless microphone used for home applications can also become a small secondary device with small footprint. The heterogeneity and coexistence characteristics of TVWS band give many challenges in order to improve the spectrum utilization effectively.

Interference mitigation in the TVWS is a challenging issue due to the limited channel availability and overlapping of multiple network coverage. The present heterogeneous network uses the ISM band at $2.4 \mathrm{GHz}$ where the interference mitigation is a serious issue [16]. The similar interference problem exists in TVWS with new interference situations such as interference between IEEE 802.11af and Ecma 392 with low power and higher power fixed systems (e.g. 802.22). Due to larger area of transmission in TVWS network, the interference at secondary receivers is very high.

Typically, in such networks two types of interference exists [17]

i) Interference due to multiple secondary heterogeneous networks

ii) Interference to/from Primary networks

The interference due to multiple secondary occurs due to selection of same TV channel by multiple CWNs because of uncoordinated selection process or limited availability. This type of interference may occur due to different transmission powers, channel bandwidth and insufficient Signal to Noise Ratio (SNR). The interference to/from Primary networks is due to interference from high TV stations transmitting from 20 to $1000 \mathrm{KW}$ to secondary network or the interference created by high power secondary transmitter to primary receiver.

The interference temperature defined in [18] gives the interference level protection from primary users at secondary receivers and is given as

$$
\mathrm{T}_{\mathrm{I}}\left(f_{c}, B\right)=\frac{\mathrm{P}_{\mathrm{I}}\left(f_{c}, B\right)}{\mathrm{kB}}
$$

Here, $\mathrm{P}_{\mathrm{I}}\left(f_{c}, B\right)$ represents the interference power for frequency $f_{c}$ over the bandwidth $B$ and $\mathrm{k}$ is the Boltzmann constant. The concept of the interference temperature is further exploited in [19].

The interference mitigation in CRN for both primary and secondary users can be carried out by interference avoidance and interference control techniques. In interference avoidance, the secondary network has to find the possible spectrum holes for coexistence. But for coexistence primary and secondary users need to set some limits on interference they caused to each other in order to guarantee QoS (Quality of Service) parameters. 
In literature the interference caused to primary user by secondary transceivers has been considered for the investigation. However, the investigation on interference characterization and mitigation at the secondary receiver due to primary and multiple secondary transmitters are not addressed significantly. Interference caused due to multiple secondary devices is a challenging issue with increase in the number of secondary users or networks. This problem becomes worse in areas where multiple secondary users select the same channel or they are working on adjacent channels.

The interference mitigation in heterogeneous CRN network can be also ensured by spectrum sharing of different wireless technologies. In open spectrum sharing heterogeneous systems coexist without centralized control as defined in [20] and interference mitigation is carried out by spectrum access etiquette. In second type of hierarchical spectrum sharing, secondary users are allowed to use the spectrum shared by primary users by ensuring the interference threshold of primary network. In order to characterize the interference, the channel state information between primary and secondary transceivers must be known or can be estimated [21].

The spatial diversity concept used in multiple-input-multiple-output (MIMO) system can also be considered by using smart antenna technology to avoid interference in heterogeneous CRN for secondary users as presented in [22]. The use of antenna selection concept in MIMO is presented for interference mitigation of secondary CRN users in [23]. The characterization of aggregate interference generated due to multiple secondary networks at the desired secondary receiver is the most important concern from QoS point of view.

\section{AGGREGATE INTERFERENCE IN HETEROGENEOUS CRN NETWORKS}

In order to improve the performance of secondary receiver in heterogeneous CRN, it is very essential to calculate the aggregate interference received at the secondary receiver. This aggregate interference is the interference generated due to multiple secondary transmitters trying to work on same frequency band allocated to primary in underlay mode of operation. It is assumed here that the interference generated due to primary transmitter is negligible. The aggregate interference is calculated by considering the spatial stochastic interference model proposed in [23].

The spatial stochastic model presented here consists of different nodes with multiple Ad-hoc and distributed network which randomly placed in a finite Euclidean space as shown in Fig. 2

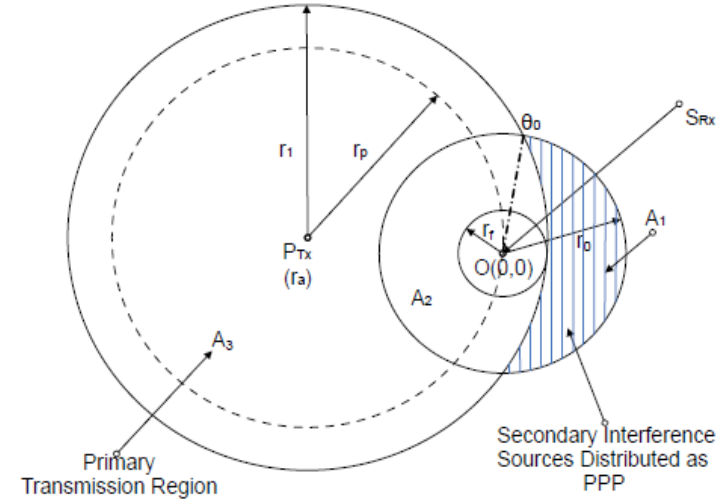

Fig. 2 Interference Model for Heterogeneous Cognitive Radio Network using TVWS

The heterogeneous CRN is considered here with primary systems operating on TVWS where all primary receivers are passive. The spatial stochastic model is considered here with $P_{T_{x}}$ as the primary transmitter having primary transmission region denoted by $A_{3}$ with radius of $r_{1}$ and is centered at $r_{\alpha}$. In spatial stochastic model it is assumed that all secondary nodes are distributed spatially as homogeneous Poison Point Process (PPP). It is further assumed that the secondary transmitter is located on the boundary of the interference region with radius $r_{p}$. The region $A_{2}$ with radius of $r_{0}$ gives the coverage area of desired secondary network. A special case with multiple secondary heterogeneous networks which are distributed spatially are assumed to be in region $A_{1}$. These multiple secondary transceivers are the sources of aggregate interference at the secondary receiver. This aggregate interference is calculated by considering the transmitted power $P_{r}$ from an interference source at distance $r$ and the channel gain between them as $h_{r}$ and is given as

$$
I(z)=\sum_{r \in \varphi} P_{r} h_{r}\|r\|^{-\alpha}
$$

Where, $\alpha$ is the propagation constant and $\varphi$ is the PPP.

The aggregate interference is further represented in terms of Laplace Transform by considering distance $\mathrm{z}$ as

$$
L_{I}(s) \triangleq E\left(e^{-s I(z)}\right)
$$

By substituting the $I(z)$ from Eq. (2) and expanding as given in [4]

$$
L_{I}(s)=\exp \left[-\pi \lambda E\left(h_{r}^{\eta}\right) \Gamma(1-\eta) s^{\eta}\right]
$$

Where $\lambda$ intensity of $\mathrm{PPP}, \eta=d / \alpha$ and for $\mathbb{R}^{2}$, $\eta=2 / \alpha$.

The channel is assumed to faded by the Nakagami-m fading channel and $E\left(h_{r}{ }^{\eta}\right)$ is given as

$$
E\left(h_{r}{ }^{\eta}\right)=\frac{\Gamma(m+\eta)}{m^{\eta} \Gamma(m)}
$$


By substituting Eq. (5) in Eq. (4),

$L_{I}(s)=\exp \left[-\pi \lambda \frac{\Gamma(m+\eta)}{m^{\eta} \Gamma(m)} \Gamma(1-\eta) s^{\eta}\right]$

By taking the inverse Laplace Transform, the PDF of aggregate interference is obtained as

$$
f_{r}(r)=\frac{\mathrm{ae}^{-a^{2} / 4 r}}{2 \sqrt{\pi} r^{\frac{3}{2}}}
$$

Here, $a=2 \pi \lambda \theta_{1} \Gamma(1+\eta)\left[\Gamma\left(\eta, r_{0}\right)-\Gamma\left(\eta, r_{1}\right)\right.$

From Eq. (7), the average Signal to Interference Noise Ratio (SINR) for this network model is given as

$$
\text { SINR }=\frac{E\left(\left|h_{i, j}\right|^{2}\right) E_{r}}{N_{0}+\operatorname{var}\left(f_{r}(r)\right)}
$$

Here, $h_{i, j}$ is the channel gain between $i^{\text {th }}$ transmitter and $j^{\text {th }}$ receiver, $E_{r}$ is the received channel gain and $N_{0}$ is PDF of the Additive White Gaussian Noise.

Further to $\operatorname{var}\left(f_{r}(r)\right)$ is calculated as

$$
\mathrm{E}\left(f_{r}(r)\right)=\int_{0}^{\infty} r f_{r}(r) d r
$$

As $r$ is tending towards $\infty$, there for the integral do not have finite value, there for it is assumed further that $r$ has finite value in the range of $0 \leq r \leq 10$, the value of $\mathrm{E}\left(f_{r}(r)\right)=-5.56 \mathrm{~dB} \quad$ and the $\operatorname{var}\left(f_{r}(r)\right)=3.8 d B$. These values are used to do the calculation of SINR presented in Eq. (8).

\section{PROPOSED MIMO BASED HETEROGENEOUS COGNITIVE RADIO NETWORK}

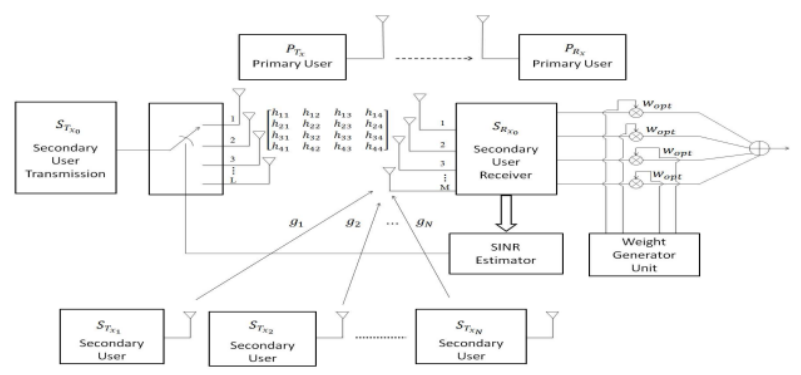

Fig. 3 Proposed MIMO based Heterogeneous Cognitive Radio Network

The interference mitigation for heterogeneous CRN is carried out by using spatial diversity based MIMO system model as shown in Fig. 3. Here the upper part of the Fig. 3 shows symbolic representation of primary transmitter $P_{T_{x}}$ and primary receiver $P_{R_{x}}$. The secondary system is equipped with multiple transmitting and receiving antennas, therefore referred as MIMO system. The secondary receiver is using optimum combining technique to estimate the received SINR and for detection of the received signal. In a heterogeneous CRN, the multiple interfering secondary nodes which are represented as $\mathrm{S}_{\mathrm{T}_{\mathrm{x}_{\mathrm{j}}}} ; \mathrm{j}=(1,2, \ldots \mathrm{N})$.These multiple interference sources are generating the aggregate interference as given by Eq. (2).

The BER analysis for the proposed system is carried out by assuming that the receiver is using coherent detection of $\mathrm{M}$-ary PSK. It is also assumed that the interference generated by all the interfering sources is equal for the simplification of mathematical expression. The maximum instantaneous SINR is given by Eq. (8). Further the average Bit Error Rate (BER) of the proposed system is calculated by considering the approach presented in [24].

The average BER for coherent BPSK can be obtained in terms of Q function as

$$
\begin{gathered}
\mathrm{P}_{\mathrm{b}}(\mathrm{E}) \simeq \\
\int_{0}^{\infty} \mathrm{Q}\left(\sqrt{2 \gamma_{\mathrm{t}}}\right) \mathrm{P}_{\gamma_{\mathrm{i}}}\left(\gamma_{\mathrm{t}} \mid \lambda_{\mathrm{n}}\right) \mathrm{d} \gamma_{\mathrm{t}}
\end{gathered}
$$

By using MGF approach the conditional BER is given in terms of MGF as

$$
\begin{array}{r}
\mathrm{P}_{\mathrm{b}}\left(\mathrm{E} \mid \lambda_{\mathrm{n}}\right)= \\
\frac{1}{\pi} \int_{0}^{\pi / 2} \mathrm{M}_{\gamma_{\mathrm{i}} \mid \lambda_{\mathrm{n}}}\left(-\frac{1}{\sin ^{2} \theta}\right) \mathrm{d} \theta
\end{array}
$$

From this the average BER for TAS/OC in multiple interference by using MGF based approach can be given for Nakagami-m fading channel as

$$
\begin{gathered}
\mathrm{P}_{\mathrm{b}}(\mathrm{E})= \\
\frac{1}{\pi} \int_{0}^{\pi / 2}\left(\frac{\sin ^{2} \theta}{\sin ^{2} \theta+\left(\overline{\gamma_{d}} / \mathrm{m}\right) /\left(1+\mathrm{M} \bar{\gamma}_{1}\right)}\right)^{\mathrm{mN}}\left(\frac{\sin ^{2} \theta}{\sin ^{2} \theta+\left(\overline{\gamma_{d}} / \mathrm{m}\right)}\right)^{\mathrm{m}(\mathrm{M}-\mathrm{N})}
\end{gathered}
$$

The closed-form solution of Eq. (12) can be obtained for $\mathrm{N}$ interferer as per [21] in case of Nakagami-m fading as given below

$$
\begin{aligned}
& \mathrm{P}_{\mathrm{b}}(\mathrm{E})= \\
& \frac{\left(1+\mathrm{M} \overline{\mathrm{Y}}_{\mathrm{L}}\right)^{\mathrm{m}(\mathrm{N}-1)}}{2\left(-\mathrm{M} \overline{\mathrm{Y}}_{\mathrm{L}}\right)^{\mathrm{M}-1}}\left[\sum_{\mathrm{k}=0}^{\mathrm{N}-1}\left(\frac{-\mathrm{M} \overline{\mathrm{Y}_{\mathrm{L}}}}{1+\mathrm{M} \overline{\mathrm{Y}}_{\mathrm{L}}}\right)^{\mathrm{k}} \mathrm{B}_{\mathrm{k}} \mathrm{I}_{\mathrm{k}}\left(\frac{\overline{\mathrm{Y}_{\mathrm{d}}} / \mathrm{m}}{1+\mathrm{M} \overline{\mathrm{Y}}_{\mathrm{L}}}\right)-\right. \\
& \left.\left(1+\mathrm{M} \overline{\gamma_{1}}\right) \sum_{\mathrm{k}=0}^{\mathrm{M}-\mathrm{N}-1}\left(-\mathrm{M}_{\overline{\gamma_{1}}}\right)^{\mathrm{k}} \mathrm{C}_{\mathrm{k}} \mathrm{I}_{\mathrm{k}}\left(\overline{\gamma_{\mathrm{d}}} / \mathrm{m}\right)\right]
\end{aligned}
$$

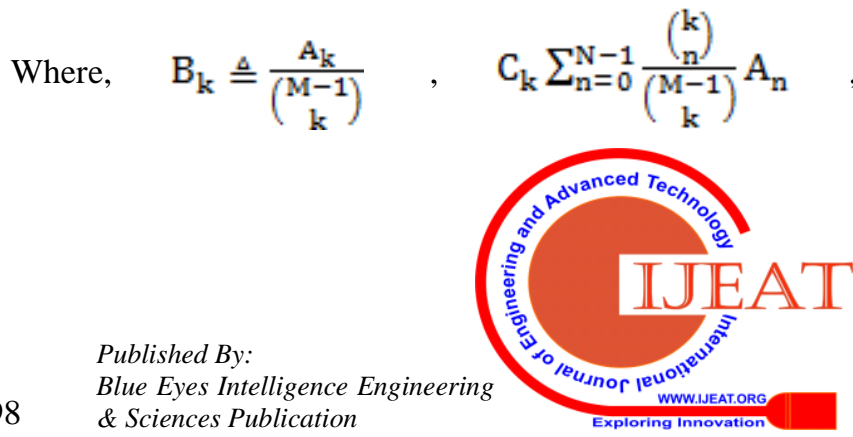




$$
\begin{aligned}
& \mathrm{A}_{\mathrm{k}} \triangleq(-1)^{\mathrm{N}-1+\mathrm{k}} \frac{\left(\begin{array}{c}
\mathrm{N}-1 \\
\mathrm{k}
\end{array}\right)}{(\mathrm{N}-1) !} \Pi_{\mathrm{n}=1}^{\mathrm{N}}(\mathrm{M}-\mathrm{n}) \text { and } \\
& \frac{1}{\mathrm{k} !} \int_{0}^{\infty} \mathrm{x}^{\mathrm{k}} \operatorname{erfc}(\sqrt{\mathrm{c} \mathrm{x}}) \mathrm{e}^{-\mathrm{x}} \mathrm{dx}
\end{aligned}
$$

Here $\mathrm{k}=0,1,2, \ldots . \mathrm{M}-2$.

\section{RESULTS AND DISCUSSION}

The performance analysis of heterogeneous CRN with MIMO is carried out for secondary transceiver by considering the multiple coexistence secondary interference sources in this section. The image data is transmitted from the secondary transmitter to the desired secondary receiver in presence of possible multiple secondary interference sources. The channel between this secondary transceiver as well as the co-channel interference model is assumed to be faded by Nakagami-m fading. The BER performance analysis presented in section 4 is verified by using Monte Carlo simulations. The aggregate interference is calculated by using Eq. (2) and the corresponding SINR by using Eq. (8) for the analysis.

The MIMO system with antenna selection is used to transmit the image data for performance analysis. The antenna which provides maximum SINR at the secondary receiver under the aggregate interference generated by multiple secondary coexistence networks is selected for transmission. The aggregate interference is calculated by considering the finite number of secondary interference sources such as IEEE 802.11, IEEE 802.22 and small interference source from wireless microphone.

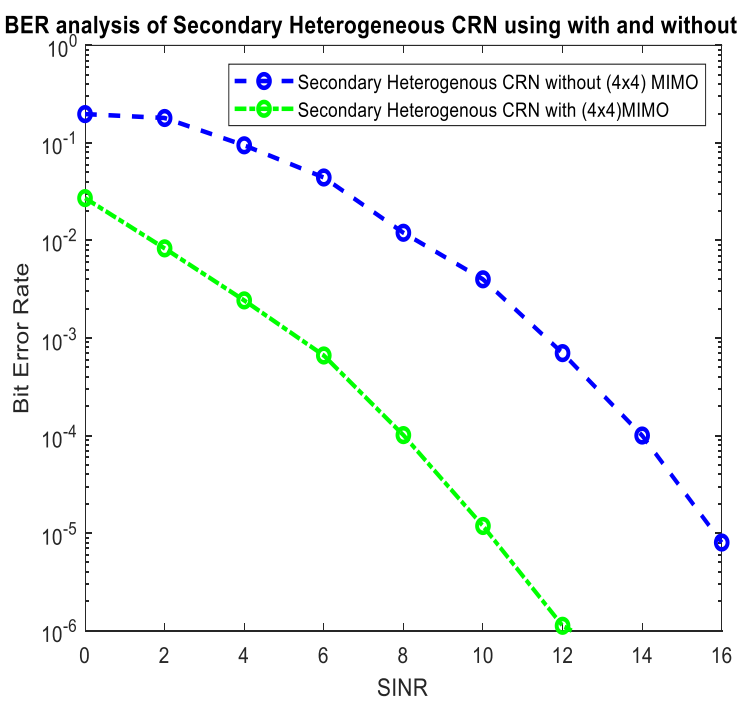

Fig. 4 BER analysis of secondary heterogeneous network using with and without (4x4) MIMO

Fig. 4 shows the BER analysis of secondary heterogeneous using with and without MIMO under aggregate interference from $\mathrm{N}=4$ interference sources. It is observed from the figure that under the impact of aggregate interference the performance of secondary network improves with (4 x 4) MIMO as compared to single antenna transmission. The number of interference sources are assumed to be $\mathrm{N}=4$ for the analysis. The BER for $(4 \times 4)$ MIMO at $6 \mathrm{~dB}$ is 0.00243 as compared to without MIMO case as 0.0946. It confirms the use of spatial diversity systems for secondary heterogeneous CRN network for performance improvement.

Fig. 5 shows the performance analysis of the proposed system with $(4 \mathrm{x} 4)$ MIMO configuration for different interference scenarios. It is observed from the figure that the BER performance of the proposed system decreases with increase in number of interference sources. The MIMO configuration with single antenna selection is considered here where, the antenna subset is selected which maximizes the received SINR and proportionally minimizes the BER at the receiver end under the impact of aggregate interference. The BER at $6 \mathrm{~dB}$ for $\mathrm{N}=1$ is 0.00243 whereas for $\mathrm{N}=4$ it is 0.0946 . Due to use of MIMO with antenna selection, the performance of secondary system is still better even by considering four different interference sources.

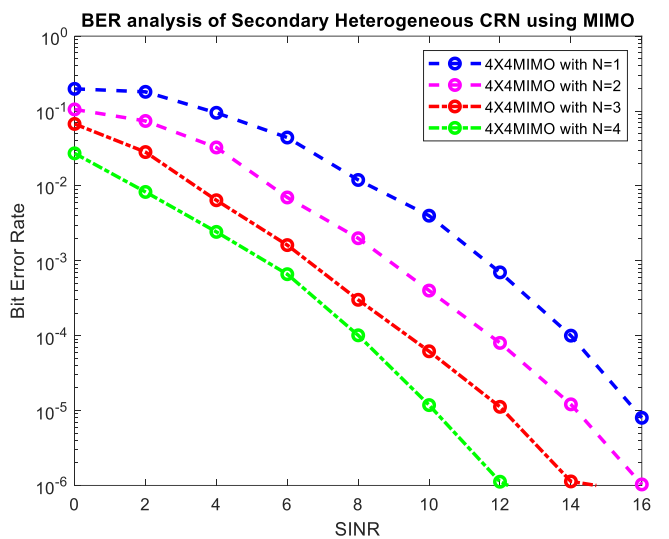

Fig. 5 BER analysis of secondary heterogeneous CRN using MIMO for $\mathrm{N}=1,2,3,4$

Fig. 6 shows the performance comparison for proposed system for two different interference scenarios. In first case, the finite number of interference sources are assumed to be finite i.e. $\mathrm{N}=4$. In second case, the number of interference sources is assumed to be large and spatially distributed using PPP as shown in model presented in section 3. It is observed that the performance of the proposed system is better even in presence of large number of interference sources.

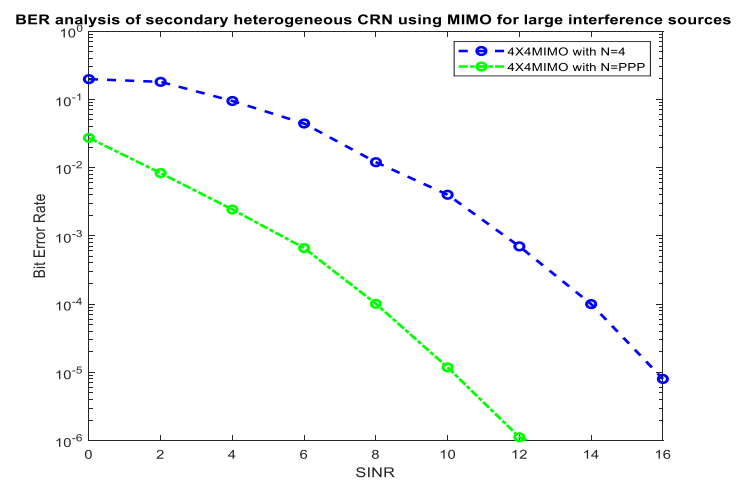

Fig. 6 BER analysis of secondary heterogeneous CRN using MIMO for large interference sources 


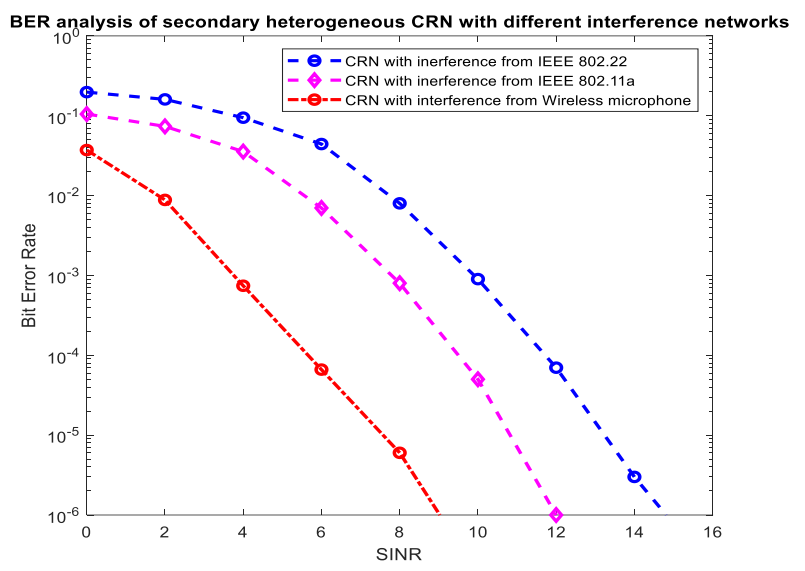

Fig. 7BER analysis of secondary heterogeneous CRN with different interference networks

The performance analysis of proposed system is carried out by considering different interference networks as shown in Fig.7. The interference networks are considered as per the model shown in Fig.1. The wireless microphone which is placed very near to the desired secondary network creates a little disturbance as compared to IEEE 802.11a network. The BER performance of proposed network degrades significantly for IEEE 802.22 network compared to rest of networks. It is assumed here that all the networks are operating in same frequency band and generates equal power but spatially located at different locations. In real life scenario, the impact can be also analyzed for different frequency bands and for different transmission power also.

\section{CONCLUSION}

In this paper, the heterogeneous cognitive radio network over TVWS is presented at physical layer. The interference mitigation problem of such a network is addressed. The spatial diversity based techniques using single antenna selection in MIMO are proposed to mitigate the interference in heterogeneous CRN. The coexistence of different wireless networks in secondary CRN is considered for analysis. The characterization of aggregate interference is carried out for secondary networks by considering different interference scenarios. The proposed heterogeneous CRN using MIMO system out performs in the presence of different interference scenarios. It is observed that the use of spatial diversity system enhances the performance of secondary system in multiple interference scenarios. The proposed system outperform even in the large number of interference sources spatially distributed as PPP. The BER performance comparison is also presented for different interference scenario such as IEEE 802.11a, IEEE 802.22 and private wireless networks also.

\section{ACKNOWLEDGMENT}

First and foremost, my appreciation and thanks go to my parents and family for their commitment, sacrifice, and overalls, their consistent encouragement and support. I wish to express my sincere thanks and gratitude to my Ph.D. guide Dr. A. K. Kureshi for his continuous guidance and help to complete this research paper. I wish to express my gratitude to the Director, National Institute of Electronics \& Information Technology, Dr. Babasaheb Ambedkar
Marathwada University, Aurangabad for their continuous support and encouragement to complete these research program requirements.

\section{REFERENCES}

1. C. Ghosh, S. Roy, and D.Cavalcanti, "Coexistence challenges for heterogeneous cognitive wireless networks in TV white spaces,"IEEE Wireless Communications Magazine, vol. 18, no. 4, pp. 22-31, 2011.

2. Lars Berlemann et. al., "Coexistence and Interworking of IEEE 802.16 and IEEE 802.11(e)", IEEE 63rd Vehicular Technology Conference, pp. 27-31, 2006

3. J. Mitola and G.Q.Maguire, "Cognitive radio: making softwwre radios more personal," IEEE Personal Communicatios, vol. 6, no. 4, pp. 13-18, August 1999.

4. M. Deshmukh, K. Patif, F. Frederiksen, K. Skouby, and R.Prasad, "Wireless broadband network on TVWS for rural areas: An Indian perspective," in Proceedings of $16^{\text {th }}$ International Symposium on Wireless Personal Multimedia Communications, WPMC June 2013.

5. J. Wang, et. al., "First Cognitive Radio Networking Standard for Personal/Portable Devices in TV White Spaces," IEEE Symposium on New Frontiers in Dynamic Spectrum (DySpan), vol.8, pp.1-12, April 2010.

6. Hëna Maloku, Zana Limani Fazliu , and Mimoza Ibrani , "A Survey on Coexistence in Heterogeneous WirelessNetworks in TV White Spaces", Wireless Communications and Mobile Computing, vol. 2018, pp. 1-14, 2018.

7. Thomas N.J.et. al.“Analysis of Co-existence between IEEE 802.11 and IEEE 802.16 Systems", in proc. Appleton Lab, Chilton vol.2, 2012

8. Xiang peng Jing and Dipankar Raychaudhuri, "Spectrum Co-existence to IEEE802.11b and 802.16a Networks Using Reactive and Proactive Etiquette Policies", in proc. Wireless information Network Laboratory (WINLAB), Rutgers University, North Brunswick, NJ. 2009.

9. Kaigui Bian et.al., "Heterogeneous Coexistence of Cognitive Radio Networks in TV White Space", arXiv:1902.06035 , Feb 2019.

10. Xiang peng Jingetal., "Reactive Cognitive Radio Algorithms for Co-Existence between IEEE802.11b and 802.16a Networks", in proc. WINLAB Rutgers University, Piscataway.

11. C. Ghosh, S. Roy,and D.Cavalcanti,"Coexistence Challenges for Heterogeneous Cognitive Wireless Networks in TV White Spaces", in proc. Electrical Engineering Department, University of Washington, North America 2018.

12. Berlemann et al." Coexistence of IEEE802.11 and IEEE802.16(a ) in Unlicensed Frequency Bands", in proc. RWTH Aachen University, Germany,2006.

13. Bo Gao, et.al.,"A Taxonomy of Coexistence Mechanisms for Heterogeneous Cognitive Radio Networks Operating in TV White Spaces ", in proc. Bradley Department of Electrical and Computer Engineering, Virginia Tech, Blacksburg, VA24061, Department of Electrical Engineering, University of Washington, 2012.

14. Bingxuan Zhao and Shigenobu Sasaki, "Spectrum Sensing for Cognitive Coexistent Heterogeneous Networks", International Journal of Distributed Sensor Networks, 2013.

15. Hesham ElSawy, Ekram Hossain, and Martin Haenggi,"Stochastic Geometry for Modeling, Analysis, and Design of Multi-Tier and Cognitive Cellular Wireless Networks : A Survey" IEEE Communications Survey and Tutorials, vol.15, no.3, 2013.

16. T. Henderson, G. Pei, R. Groves, T. Bosaw, M. Rush, C. Ghosh, and S Roy, "Wireless Network Coexistence," Boeing Project Report, Dec. 17, 2009.

17. Ayaskanta Mishra, "Co-existence Issue in IoT Deployment using Heterogeneous WirelessNetwork (HetNet): Interference Mitigation using Cognitive Radio", International Journal on Advanced Science, Engineering Information Technology, vol. 9, no. 1, pp. 109-120,2019.

18. Establishment of interference temperature metric to quantify and manage interference and to expand available unlicensedoperation in certain xed mobile and satellite frequency bands, “ FCC, 2003, FCC Doc. ET Docket 03-289

19. T. C. Clancy, "On the use of interference temperature for dynamic spectrum access," in Annals of Telecommunications, vol. 64, no. 7, pp. 573-585, Springer, 2009 
20. J. Lee, H. Wang, S. Kim, and D. Hong, "Sensing thresholdcontrol for fair coexistence of heterogeneous systems in openspectrum," IEEE Transactions on Wireless Communications, vol.8, no. 12, pp. 5773-5778, 2009.

21. V. Gardellin, S. Das, and L. Lenzini, "Self-coexistence in cellularcognitive radio networks based on the IEEE 802.22 standard,'IEEEWireless CommunicationsMagazine, vol. 20, no. 2, pp. 52-59, 2013.

22. R. Zhang and , Y. Liang, "Exploiting Multi-Antennas for Opportunistic Spectrum Sharing in Cognitive Radio Networks," IEEE J. of Selected Topics in Signal Processing, vol. 02, no. 01, pp. 88-102, Feb. 2008.

23. Vaibhav Hendre et. al., " Transmit Antenna Selection with Optimum Combining for Aggregate Interference in Cognitive Underlay RadioNetwork", Wireless Personal Communication, Springer, vol. 92, no.34, pp. 1071-1088, February2017.

24. Simon M. K. and Alouni M. S. (2005). Digital Communication over fading channels. 2nd ed. John Wiely \& Sons

\section{AUTHORS PROFILE}

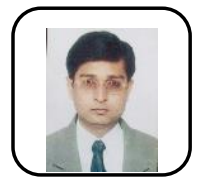

Ravindra H. Adekar is a Research Scholar in National Institute of Electronics \& Information Technology, Dr. Babasaheb Ambedkar Marathwada University, Aurangabad, India. He is presently working as an Assistant Professor in Computer Science and Engineering Department, Shri Tulja Bhavani College of Engineering, Tuljapur, India. He has completed Bachelor of Engineering (BE) in Electronics from Dr. Babasaheb Ambedkar Marathwada University, Aurangabad, India, Master of Engineering (ME)) in Electronics (Digital Systems) from University of Pune, India. He is currently a Ph.D. candidate and Research Scholar in National Institute of Electronics \& Information Technology, Dr. Babasaheb Ambedkar Marathwada University, Aurangabad, India. His research interest includes analysis, and optimization of wireless/mobile communications networks, cognitive radio technologies. He has published total of 05 research papers in International/National Journals and International/National Conferences. He has guided total of 12 under-graduate projects. He is a Member of LMIETE. Email: rhadekar@gmail.com

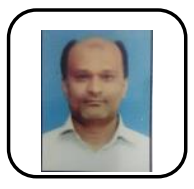

Dr. A. K. Kureshi is a Professor in the Department of Electronics and Telecommunication Engineering and is also a Director of MMANTC, College of Engineering MANSOORA, Malegaon under Savitribai Phule Pune University, India. He has completed Bachelor of Engineering (BE) in Electronics, Master of Engineering (ME)) in Electronics and He received his Ph.D. in VLSI Design from Aligarh Muslim University, Aligarh, India, in 2010. His research interest includes VLSI design, analysis, and optimization of wireless/mobile communications networks, cognitive radio technologies. He has published total of 35 research papers in International/National Journals and International/National Conferences. He has guided total of 70 under-graduate projects. He is presently working on low power architecture for FPGAs a research project sanction by Savitribai Phule Pune University under QIP. He is Member of LMISTE, FIE, IEEE, CMI (UK). He has received Best paper award at International Conference by Pentagram research center Hyderabad and Engineering Achievement award by Institution of Engineers Ahmednagar local centre. Email: akkureshi@rediffmail.com 